Clío América/ Vol. 12, No. 24 - 2018 / 149 - 162

DOI: http://dx:10.21676/23897848.2864

\title{
PERSONALIDAD DE MARCA EN INSTITUCIONES DE EDUCACIÓN SUPERIOR DEL NOROESTE DE MÉXICO
}

\author{
BRAND PERSONALITY OF HIGHER EDUCATION INSTITUTIONS \\ IN THE NORTHWEST OF MEXICO
}

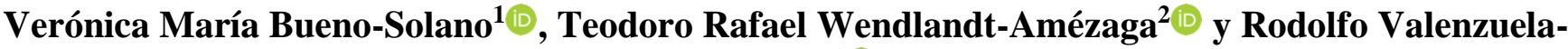 Reynaga $^{3}$ iD}

Tipología: Artículo de investigación científica y tecnológica

Para citar este artículo: Bueno, V. M., Wendlandt, T. R. y Valenzuela, R. R. (2018). Personalidad de marca en instituciones de educación superior en un municipio del noroeste de México. Clío América, 12(24), 149 - 162. doi: http://dx.org/10.21676/23897848.2864

Recibido en septiembre 07 de 2018

Aceptado en 12 de noviembre de 2018

Publicado en línea en 28 de noviembre de 2018

RESUMEN

El logotipo es un elemento de marca que favorece a la identificación de las empresas por parte de los consumidores. En este sentido, la presente investigación propone medir la percepción de la personalidad de marca de tres instituciones de educación superior en el municipio de Cajeme, al sur del estado de Sonora en México, para ello se utiliza una metodología cuantitativa de tipo descriptiva-correlativa, mediante un diseño no experimental. La información se recopiló mediante un instrumento de medición basado en el modelo de personalidad de marca de Jennifer L. Aaker y como parte de los hallazgos, se identificaron ocho dimensiones de personalidad de marca; Competencia, Sinceridad, Entusiasmo, Sofisticación, Delicadeza, Rudeza, Regionalismo, Cooperación, de las cuales las dimensiones de Sinceridad y Delicadeza mostraron cierta asociación con el Instituto Tecnológico de Sonora, Sofisticación y Cooperación con la Universidad La Salle Noroeste, y Entusiasmo con el Instituto Tecnológico Superior de Cajeme. Finalmente, se proyectó una posición ideal donde la dimensión de Competencia fue la mejor valorada. La utilidad del estudio radica en la posibilidad de mejorar la gestión de la personalidad de marca institucional, a efecto de lograr un reposicionamiento estratégico positivo.

Palabras clave: personalidad de marca - institución - educación superior - percepción.

JEL: M3; M30; M31; M32.

\section{ABSTRACT}

A logotype is an element of mark that allows consumers to identify a company. In this regard, the present investigation proposes to measure the perception of the brand personality of three institutions of higher education in the Municipality of Cajeme, South of the State of Sonora, in Mexico. For this purpose, a quantitative methodology of descriptive-correlative type is used, by means of a non-experimental design. The information was compiled using a measuring instrument based on Jennifer L. Aaker's brand personality framework, and, as part of the findings, eight dimensions of brand personality were identified (Competence, Sincerity, Enthusiasm, Sophistication, Delicacy, Rudeness, Regionalism, Cooperation). The dimensions of Sincerity and Delicacy were shown to have certain association with the Sonora Institute of Technology, Sophistication and Cooperation with the La SalleUniversity of the Northwest, and Enthusiasm with the Cajeme Higher Institute of Technology. Finally, an ideal situation was projected, in which the dimension of Competence was the best valued. The usefulness of the study lies in the possibility of improving management of institutional brand personality, in order to achieve positive strategic repositioning.

Keywords: brand personality - institution - higher education - perception.

1 Departamento de Administración, Instituto Tecnológico de Sonora. Ciudad Obregón, Sonora, México. Email: veronicabuenosolano@gmail.com ORCID: http://orcid.org/0000-0001-7370-4736

2 Departamento de Administración, Instituto Tecnológico de Sonora. Ciudad Obregón, Sonora, México. Email: teodoro.wendlandt@itson.edu.mx ORCID: http://orcid.org/0000-0003-0244-4062

3 Departamento de Contaduría y Finanzas, Instituto Tecnológico de Sonora. Ciudad Obregón, Sonora, México. Email: rodolfo.valenzuela@itson.edu.mx ORCID: http://orcid.org/0000-0003-3408-361X 


\section{INTRODUCCIÓN}

Anteriormente las marcas eran definidas a partir de las características, atributos y valores que el propietario de una empresa pretendía transmitir (Baños y Rodríguez, 2012) y mediante los diversos recursos de comunicación se conseguía que estos elementos quedaran registrados en la mente de los consumidores. Esta acción consistía básicamente en un proceso unidireccional, en el que la empresa emitía un mensaje a través de la marca y el público lo recibía. En la actualidad, son los consumidores los que deciden mediante qué rasgos reconocen y definen a las marcas en el entorno social, siendo esto conocido como personalidad de marca (Cabezas y Orellana, 2014).

Es tal la relevancia estratégica que existe al identificar y comprender la percepción de los consumidores hacia una determinada marca y los elementos que la representan (Keller, 1993), que se le ha otorgado especial atención al estudio de la personalidad de marca. En particular, este constructo es definido por Aaker (1997) como "un conjunto de características humanas asociadas a una marca" ( $p$. 347). Por lo tanto, a través de la marca es posible reunir todas las características psicológicas que se le atribuyen a un producto o servicio, independientemente de sus propiedades materiales (Lodos, 2011), siendo la imagen de marca el concepto que el público vincula con una marca específica, producto o servicio (Capriotti, 2013).

Al considerar lo anterior, la marca representa un cúmulo de rasgos -positivos o negativos- que invariablemente ejercerán influencia en la decisión de compra de los consumidores (Aaker, 1999; Llopis, 2015). Esto se debe al fenómeno de que al identificarse con la marca -aun cuando no haya tenido una experiencia previa con ella- el consumidor podría otorgarle cierta personalidad a la misma debido a la necesidad de reafirmar su individualidad $\mathrm{y}$, por consecuencia, opte por una alternativa que sea afín a sí mismo (Gómez, 2010; Kleine, Kleine y Allen, 1995; Malhotra, 1988).

Esto es de gran importancia para las organizaciones, ya que a partir del estudio de la personalidad de marca es posible encaminar los/sus esfuerzos para entender las preferencias del público, lo anterior con el objeto de lograr adaptarse a la personalidad del individuo en sí y reforzar el vínculo existente (Schlesinger y Cervera, 2008). Al respecto, Pirela, Villavicencio y Saavedra (2004) señalan que percatarse y dominar los atributos de la personalidad de marca propicia la creación de una relación leal entre esta y el consumidor, lo que a su vez representa un vínculo entre los rasgos de personalidad humanos con los de la marca.

En un proceso adecuado de gestión de marca, esta posee un papel fundamental al ser parte de su posicionamiento e imagen, además que provee apoyo en el desarrollo del valor de marca y resulta útil al otorgarle reconocimiento de carácter global (Plummer, 2000). Entre otras, una de las principales ventajas es la identificación y diferenciación de la empresa mediante la creación de vínculos de carácter emocional con el consumidor, lo que adicionalmente representa un medio propio para la proyección corporativa (Benavides, 2012). Eventualmente, esta proyección favorecerá la reputación de la empresa y logrará crear cierto reconocimiento social.

Por otro lado, un elemento de marca que contribuye en gran medida al reconocimiento de esta es el logotipo. Según Kotler y Armstrong (2013), este simbolismo gráfico puede respaldar el posicionamiento de una marca, así como fortalecer su personalidad, de esta manera, el logotipo por sí solo produce ciertas apreciaciones en el consumidor, que a su vez pueden ser independientes de las generadas por la marca en sí. Cabe señalar que el logotipo es un elemento que se encuentra presente en las empresas como parte de su identidad corporativa y es uno de los componentes de la comunicación institucional, llegando a tener un valor fundamental e intangible en las organizaciones.

De acuerdo con Cussi (2016), el logotipo funge como un signo distintivo que permite diferenciar ciertos productos o servicios en el mercado, además que la personalidad de marca puede ser expresada $y$ 
percibida en función de ese símbolo gráfico, por lo que esta representa un intangible que, al mismo tiempo, se apoya de recursos tangibles como el logotipo; el cual es probablemente uno de los canales principales a través del cual las organizaciones transmiten su personalidad de marca.

Partiendo de la importancia de una adecuada gestión de la marca institucional, el presente estudio se propuso medir la percepción de la personalidad de marca de tres instituciones de educación superior en el municipio de Cajeme, al sur del estado de Sonora en México. Es pertinente mencionar que, durante los últimos años, en esta localidad se ha incrementado considerablemente el número de universidades, tanto públicas como privadas, de acuerdo con el Directorio Estadístico Nacional de Unidades Económicas (DENUE) del Instituto Nacional de Estadística y Geografía (INEGI). No obstante, es posible identificar tres instituciones de educación superior que cuentan con una mayor cantidad de años en esta entidad territorial, siendo estas: el Instituto Tecnológico de Sonora (ITSON), la Universidad La Salle Noroeste (ULSA) y el Instituto Tecnológico Superior de Cajeme (ITESCA).

Para cumplir con el objetivo planteado, se pretende analizar y comparar la percepción sobre las dimensiones y rasgos de la personalidad de la marca de cada una de las instituciones educativas en estudio, utilizando para ello el logotipo correspondiente. Cabe mencionar que se empleará el modelo de medición propuesto por Aaker (1997), considerando la modificación del instrumento sugerida por Álvarez y Harris (2002), esto con la finalidad de obtener un resultado más preciso al utilizar las dimensiones que mejor se adapten a la cultura mexicana.

El presente documento se organiza por un apartado de antecedentes, en donde se describen estudios empíricos que abordan la personalidad de marca, posteriormente se presentan las características metodológicas de la investigación, entre las que se encuentran; los atributos de los sujetos participantes, el diseño y desarrollo del instrumento de medición, las técnicas de análisis de datos utilizadas y el procedimiento general de cada una de las etapas de la investigación. En seguida se muestran los hallazgos del estudio, dividido en los apartados de rasgos, dimensiones y situación ideal, finalmente, se discuten cada uno de los resultados y se proporcionan algunas aseveraciones a manera de conclusión, incluyéndose recomendaciones para estudios futuros.

\section{Antecedentes y estudios empíricos de la personalidad de marca}

En Aaker (1997), realizó un estudio en Estados Unidos donde originalmente pretendía identificar la relación entre la personalidad de los individuos y la otorgada a la marca, así como conocer en qué grado influía este factor en su decisión de compra. Para ello se fundamentó en la teoría de la personalidad humana, donde desarrolló y validó una escala que se integra por cinco dimensiones; sinceridad, emocionante, competencia, sofisticado y rudeza, las cuales miden la personalidad de marca. Dicho trabajo ha sido utilizado como referente y fundamento teórico en diversas investigaciones posteriores (Álvarez y Harris, 2002; Olavarrieta, Friedmann y Manzur, 2004; Pirela et al., 2004; Saavedra, Urdaneta, Pirela y Colmenares, 2008; Watkins y Gonzenbach, 2013), siendo en ocasiones compatibles algunas de sus dimensiones dentro de culturas y contextos distintos (Aaker, Benet y Garolera, 2001; Goñi, Torres y Aguilera, 2013; Peñaloza, et al., 2016; Sung y Tinkham, 2005).

Tal es el caso de un estudio realizado por Álvarez y Harris (2002) en México, en donde se identificó e integró una dimensión alterna denominada "género", esto al comparar algunas marcas mexicanas globales y locales. Por su parte en Venezuela, Pirela et al. (2004) llevaron a cabo una investigación exploratoria del modelo de personalidad de marca utilizando los centros comerciales como referencia, en donde a diferencia de otros estudios en Latinoamérica, las dimensiones que se caracterizaron fueron emocionante, sinceridad, pasional, pasividad y rudeza; cabe mencionar que ciertas dimensiones se comparan con los hallazgos encontrados por Saavedra et al. (2008), quienes midieron la 
personalidad de marca en el sector automotriz venezolano y concluyeron que las dimensiones más destacadas fueron emocionante, pacífico, pasional y sincero, pudiendo constatar la concordancia en la mayoría de las dimensiones entre ambos estudios.

Por último, en una investigación realizada por Goñi et al. (2013) se identificaron las dimensiones y los rasgos de la personalidad de marca en México, y se compararon con aquellas dimensiones obtenidas en estudios previos en Estados Unidos y Chile. Los resultados se mostraron consistentes con los de Aaker (1997) en Estados Unidos, aunque se agregó una dimensión adicional para México (espontaneidad) y otra para Chile (tradicional); siendo el resto de las dimensiones similares para los tres países. Según los autores del estudio, las diferencias mostradas en el mismo posiblemente se deban a variables culturales no consideradas, por lo que cada nueva dimensión podría ser atribuible a aspectos culturales cada país.

En la actualidad las instituciones educativas buscan diseñar y planear estrategias de mercadotecnia efectivas, las cuales dan a conocer sus atributos distintivos a la sociedad en general, principalmente a los aspirantes universitarios (Arpan, Raney y Zivnuska, 2003). La imagen corporativa de estas instituciones, específicamente las de educación superior, es parte fundamental de la personalidad de marca de las mismas, por tal motivo resulta relevante conocer la percepción de los estudiantes y aspirantes en cuanto a la personalidad de marca a través del logotipo de estas organizaciones, lo que a su vez permite la generación de estrategias eficaces para aprovechar este elemento en su beneficio.

\section{METODOLOGÍA}

La metodología utilizada para este estudio fue cuantitativa, a través de un diseño de investigación no experimental de corte transversal. El tipo de estudio fue descriptivo-correlativo, cualidad propia de las caracterizaciones a realizar y de las asociaciones a comprobar. Asimismo, se estableció que la población a investigar estuviese integrada por todos los estudiantes de nivel preparatoria $\mathrm{y}$ universidad del municipio de Cajeme, cuya edad mínima fuese de 15 años. Para la determinación de la muestra, se utilizó un método de muestreo no probabilístico por conveniencia debido a la dificultad que se presentó con relación a la obtención de una lista de muestreo con todos los sujetos correspondientes.

Los datos se obtuvieron de la aplicación del instrumento a una muestra ( $n$ ) conformada por 94 estudiantes, cuya edad promedio fue de 20,9 años y una desviación estándar de 7,5 años, incorporándose todas las edades dentro de un rango de 54 años desde los 15 hasta los 69 años. Además que $70(74,5 \%)$ sujetos fueron del sexo femenino y $24(25,5 \%)$ del masculino. Por último, del total de participantes 42 $(44,7 \%)$ sujetos señalaron poseer un nivel de escolaridad de preparatoria, 43 (45,7 \%) sujetos un nivel escolar de licenciatura o ingeniería, y los $9(9,6$ $\%$ ) sujetos restantes presentaron un nivel de estudios de posgrado -programas de maestría y doctorado-. Cada sujeto que participó en el estudio respondió un instrumento de medición por cada logotipo de las tres instituciones educativas, administrándose en total 282 instrumentos.

La recopilación de los datos comprendió un período de tiempo de cuatro meses, de enero a mayo de 2018, para lo cual se utilizó un instrumento integrado por dos secciones compuestas por 45 preguntas, donde el tiempo promedio estimado de respuesta fue de 15 minutos. En la primera sección se inquirió sobre las características sociodemográficas de los sujetos participantes -edad, sexo y nivel de escolaridad-, mientras que en la segunda sección se evaluaron los 42 rasgos de personalidad de marca de Aaker (1997), esto conforme a la percepción subjetiva de los sujetos sobre cada uno de los logotipos institucionales. Es importante señalar que el formato general de respuesta fue una escala tipo Likert de cinco opciones, las cuales variaban desde 1 (Nada descriptivo) hasta 5 (Muy descriptivo), siendo la puntuación más alta un indicativo de un mayor grado de representatividad percibida (Figura 1). 
Figura 1. Instrumento de medición del estudio

\section{MEDICIÓN DE LA PERSONALIDAD DE MARCA DE LOS LOGOTIPOS DE INSTITUCIONES DE EDUCACIÓN SUPERIOR EN EL MUNICIPIO DE CAJEME}

Instrucciones: El presente cuestionario tiene como objetivo medir la personalidad de marca de tres universidades mediante su percepción en relación a sus logotipos. Se pide evalúe y conteste las siguientes preguntas. Todas las respuestas serán confidenciales, siendo utilizadas para fines de investigación. El promedio de respuesta del cuestionario es de 15 minutos.

1. A continuación se le pide proporcionar los siguientes datos sociodemográficos.

a) Sexo: ( ) Femenino ( ) Masculino

b) Edad: años

c) Nivel de escolaridad: ( ) Preparatoria ( ) Licenciatura/Ingeniería ( ) Posgrado

2. Seguido se le pide evaluar y encerrar con un círculo el número que según su opinión, refleje qué tan descriptivos son los conceptos presentados en la tabla con respecto a los siguientes logotipos:
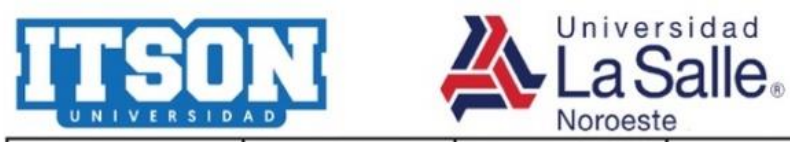

\begin{tabular}{|c|c|c|c|c|c|}
\hline & Nada descriptivo & Poco descriptivo & Indiferente & Algo descriptivo & Muy descriptivo \\
\hline Realista & 1 & 2 & 3 & 4 & 5 \\
\hline Honesto & 1 & 2 & 3 & 4 & 5 \\
\hline Saludable & 1 & 2 & 3 & 4 & 5 \\
\hline Alegre & 1 & 2 & 3 & 4 & 5 \\
\hline Atrevido & 1 & 2 & 3 & 4 & 5 \\
\hline Único & 1 & 2 & 3 & 4 & 5 \\
\hline Actual & 1 & 2 & 3 & 4 & 5 \\
\hline Enérgico & 1 & 2 & 3 & 4 & 5 \\
\hline Inteligente & 1 & 2 & 3 & 4 & 5 \\
\hline Confiable & 1 & 2 & 3 & 4 & 5 \\
\hline Exitoso & 1 & 2 & 3 & 4 & 5 \\
\hline Clase alta & 1 & 2 & 3 & 4 & 5 \\
\hline Encantador & 1 & 2 & 3 & 4 & 5 \\
\hline Rudo & 1 & 2 & 3 & 4 & 5 \\
\hline Deportivo & 1 & 2 & 3 & 4 & 5 \\
\hline Orientado a la familia & 1 & 2 & 3 & 4 & 5 \\
\hline Sincero & 1 & 2 & 3 & 4 & 5 \\
\hline Original & 1 & 2 & 3 & 4 & 5 \\
\hline Sentimental & 1 & 2 & 3 & 4 & 5 \\
\hline A la moda & 1 & 2 & 3 & 4 & 5 \\
\hline Imaginativo & 1 & 2 & 3 & 4 & 5 \\
\hline Independiente & 1 & 2 & 3 & 4 & 5 \\
\hline Sensacional & 1 & 2 & 3 & 4 & 5 \\
\hline Especializado & 1 & 2 & 3 & 4 & 5 \\
\hline Trabajador & 1 & 2 & 3 & 4 & 5 \\
\hline Lider & 1 & 2 & 3 & 4 & 5 \\
\hline Elegante & 1 & 2 & 3 & 4 & 5 \\
\hline Femenino & 1 & 2 & 3 & 4 & 5 \\
\hline Fuerte & 1 & 2 & 3 & 4 & 5 \\
\hline Masculino & 1 & 2 & 3 & 4 & 5 \\
\hline Pueblerino & 1 & 2 & 3 & 4 & 5 \\
\hline Real & 1 & 2 & 3 & 4 & 5 \\
\hline Amistoso & 1 & 2 & 3 & 4 & 5 \\
\hline Excitante & 1 & 2 & 3 & 4 & 5 \\
\hline Moderno & 1 & 2 & 3 & 4 & 5 \\
\hline Joven & 1 & 2 & 3 & 4 & 5 \\
\hline Corporativo & 1 & 2 & 3 & 4 & 5 \\
\hline Seguro & 1 & 2 & 3 & 4 & 5 \\
\hline Confiado & 1 & 2 & 3 & 4 & 5 \\
\hline Atractivo & 1 & 2 & 3 & 4 & 5 \\
\hline Tranquilo & 1 & 2 & 3 & 4 & 5 \\
\hline Norteño & 1 & 2 & 3 & 4 & 5 \\
\hline
\end{tabular}

\section{Fuente: elaboración propia.}

Se verificó la validez de constructo o estructura factorial del instrumento para la variable de personalidad de marca, para lo cual se llevó a cabo un análisis factorial exploratorio con el método de 
componentes principales y una rotación varimax. Los resultados del análisis mostraron una muy buena adecuación de los datos (Tabla 1), en donde se obtuvo un índice Kaiser-Meyer-Olkin (KMO) de 0,916, una prueba de esfericidad de Bartlett significativa reportando un estadístico chi-cuadrada de 5035,2 con un valor de 595 grados de libertad y un valor $p$ menor a 0,001 , un determinante de $7,17 \mathrm{x}$ $10^{-9}$ y valores mayores a 0,45 en todas las comunalidades. Todo lo anterior sugiere un buen ajuste de este modelo de análisis con los datos obtenidos (Hair, Anderson, Tatham, \& Black, 1999; Ho, 2006; Pett, Lackey, \& Sullivan, 2003).

Tabla 1. Resumen de ítems y cargas factoriales del análisis factorial exploratorio $(n=282)$

\begin{tabular}{|c|c|c|c|c|c|c|c|c|c|}
\hline & \multicolumn{8}{|c|}{ Cargas factoriales } & \\
\hline Ítems/Rasgos & 1 & 2 & 3 & 4 & 5 & 6 & 7 & 8 & $h^{2}$ \\
\hline Ítem 25. Trabajador & $\mathbf{0 , 7 3}$ & 0,09 & 0,17 & $-0,09$ & 0,03 & 0,19 & 0,16 & 0,11 & 0,66 \\
\hline Ítem 38. Seguro & $\mathbf{0 , 7 0}$ & 0,28 & 0,09 & 0,34 & 0,11 & 0,02 & $-0,05$ & 0,10 & 0,72 \\
\hline Ítem 24. Especializado & 0,66 & 0,17 & 0,25 & 0,05 & 0,08 & 0,25 & 0,05 & 0,02 & 0,61 \\
\hline Ítem 26. Líder & $\mathbf{0 , 6 3}$ & 0,03 & 0,20 & 0,34 & 0,20 & 0,14 & 0,05 & 0,03 & 0,62 \\
\hline Ítem 39. Confiado & $\mathbf{0 , 6 2}$ & 0,31 & 0,11 & 0,24 & 0,23 & 0,15 & $-0,10$ & 0,12 & 0,65 \\
\hline Ítem 22. Independiente & $\mathbf{0 , 5 8}$ & 0,24 & 0,40 & $-0,05$ & 0,14 & 0,14 & $-0,16$ & 0,09 & 0,63 \\
\hline Ítem 37. Corporativo & $\mathbf{0 , 5 5}$ & 0,12 & $-0,10$ & 0,35 & 0,05 & 0,17 & $-0,14$ & 0,11 & 0,51 \\
\hline Ítem 9. Inteligente & $\mathbf{0 , 5 4}$ & 0,36 & 0,31 & 0,19 & 0,13 & 0,04 & 0,03 & 0,08 & 0,58 \\
\hline Ítem 10. Confiable & $\mathbf{0 , 5 2}$ & 0,42 & 0,17 & 0,32 & 0,02 & $-0,08$ & 0,01 & 0,07 & 0,59 \\
\hline Ítem 41. Tranquilo & $\mathbf{0 , 5 0}$ & 0,21 & 0,00 & 0,17 & 0,27 & $-0,16$ & 0,19 & 0,04 & 0,46 \\
\hline Ítem 17. Sincero & 0,49 & 0,39 & 0,23 & 0,12 & 0,10 & 0,02 & 0,25 & 0,33 & 0,64 \\
\hline Ítem 2. Honesto & 0,35 & 0,71 & 0,13 & 0,17 & $-0,00$ & 0,10 & 0,14 & 0,05 & 0,70 \\
\hline Ítem 1. Realista & 0,38 & $\mathbf{0 , 6 8}$ & 0,20 & 0,10 & $-0,03$ & 0,20 & 0,04 & $-0,10$ & 0,70 \\
\hline Ítem 3. Saludable & 0,29 & $\mathbf{0 , 6 5}$ & $-0,06$ & 0,08 & 0,29 & $-0,09$ & 0,10 & 0,21 & 0,67 \\
\hline Ítem 4. Alegre & 0,04 & $\mathbf{0 , 6 3}$ & 0,21 & 0,20 & 0,45 & 0,06 & $-0,14$ & 0,20 & 0,75 \\
\hline Ítem 18. Original & 0,23 & 0,06 & 0,79 & 0,13 & 0,01 & 0,07 & 0,02 & 0,05 & 0,71 \\
\hline Ítem 6. Único & 0,13 & 0,09 & $\mathbf{0 , 7 8}$ & 0,22 & $-0,01$ & 0,08 & 0,01 & $-0,01$ & 0,69 \\
\hline Ítem 7. Actual & 0,20 & 0,26 & 0,62 & 0,36 & 0,14 & $-0,04$ & $-0,10$ & 0,07 & 0,66 \\
\hline Ítem 21. Imaginativo & 0,43 & 0,10 & $\mathbf{0 , 5 7}$ & $-0,17$ & 0,27 & 0,09 & $-0,20$ & 0,21 & 0,71 \\
\hline Ítem 12. Clase alta & 0,17 & 0,16 & 0,17 & $\mathbf{0 , 8 0}$ & 0,10 & 0,07 & $-0,06$ & 0,16 & 0,76 \\
\hline Ítem 27. Elegante & 0,39 & 0,03 & 0,17 & $\mathbf{0 , 5 8}$ & 0,31 & $-0,07$ & $-0,24$ & 0,06 & 0,68 \\
\hline Ítem 13. Encantador & 0,12 & 0,24 & 0,35 & $\mathbf{0 , 5 8}$ & 0,24 & 0,06 & 0,11 & 0,20 & 0,63 \\
\hline Ítem 11. Exitoso & 0,39 & 0,39 & 0,18 & $\mathbf{0 , 5 2}$ & 0,03 & 0,09 & $-0,02$ & 0,15 & 0,64 \\
\hline Ítem 28. Femenino & 0,02 & 0,02 & $-0,12$ & 0,19 & $\mathbf{0 , 7 2}$ & 0,19 & 0,10 & 0,02 & 0,62 \\
\hline Ítem 34. Excitante & 0,25 & 0,04 & 0,24 & 0,20 & 0,61 & 0,17 & 0,08 & 0,11 & 0,58 \\
\hline Ítem 33. Amistoso & 0,35 & 0,30 & 0,11 & 0,08 & $\mathbf{0 , 5 6}$ & $-0,07$ & 0,21 & 0,21 & 0,64 \\
\hline Ítem 19. Sentimental & 0,21 & 0,18 & 0,18 & $-0,06$ & $\mathbf{0 , 5 4}$ & $-0,04$ & 0,10 & 0,30 & 0,51 \\
\hline Ítem 14. Rudo & 0,14 & 0,01 & 0,19 & 0,03 & $-0,15$ & 0,74 & 0,08 & 0,32 & 0,74 \\
\hline Ítem 30. Masculino & 0,16 & 0,07 & $-0,18$ & $-0,01$ & 0,27 & $\mathbf{0 , 7 1}$ & 0,17 & $-0,21$ & 0,71 \\
\hline Ítem 29. Fuerte & 0,43 & 0,02 & 0,17 & 0,10 & 0,14 & 0,61 & 0,03 & 0,03 & 0,62 \\
\hline Ítem 5. Atrevido & $-0,07$ & 0,33 & 0,40 & 0,07 & 0,31 & 0,49 & $-0,17$ & 0,18 & 0,67 \\
\hline Ítem 31. Pueblerino & 0,05 & 0,07 & $-0,10$ & $-0,22$ & 0,10 & 0,01 & $\mathbf{0 , 8 2}$ & $-0,00$ & 0,74 \\
\hline Ítem 42. Norteño & $-0,00$ & 0,03 & 0,01 & 0,08 & 0,14 & 0,13 & $\mathbf{0 , 8 1}$ & 0,08 & 0,71 \\
\hline Ítem 15. Deportivo & 0,05 & 0,10 & 0,07 & 0,23 & 0,16 & 0,26 & $-0,05$ & $\mathbf{0 , 7 4}$ & 0,71 \\
\hline Ítem 16. Orientado a la familia & 0,29 & 0,12 & 0,04 & 0,15 & 0,24 & $-0,10$ & 0,13 & 0,71 & 0,71 \\
\hline
\end{tabular}

Nota: Números en negrita indican cargas factoriales elevadas. h2= comunalidad.

Fuente: elaboración propia. 
Si bien la categorización de los ítems o atributos puede proporcionar resultados muy variados (Myers y Shocker, 1981), el criterio para la inclusión de los factores en la solución factorial fue que al menos cada factor obtuviera un valor (Eigenvalue) superior a uno, además de considerar el gráfico de sedimentación (Field, 2018; Martínez, Hernández y Hernández, 2006). Lo anterior resultó en una solución integrada por ocho factores que explicaron el $65,4 \%$ de la varianza de los puntajes totales del instrumento de medición. El primer factor se integró por 11 ítems (números: 9, 10, 17, 22, 24, 25, 26, 37, 38,39 y 41) explicando un 33,34 \% de la varianza, los factores segundo (números: 1, 2, 3, 4), tercero (números: 6, 7, 18 y 21), cuarto (números: 11, 12, 13 y 27), quinto (números: 19, 28, 33 y 34) y sexto (números: 5, 14, 29 y 30) se integraron de cuatro ítems cada uno, explicando el 6,87 \%, 5,78 \%, 5,32 $\%, 4,29 \%$ y $3,49 \%$ respectivamente. Finalmente el séptimo (números: 31 y 42) y octavo (números: 15 y 16) factor se conformaron de dos ítems cada uno, explicando el 3,33\% y el 2,98\% correspondiente. Debido a que siete ítems generaban confusión en la estructura factorial y algunos presentaban cargas muy similares, fueron eliminados (números: 8, 20, $23,32,35,36,40)$ para proporcionar una mayor claridad en la solución matricial.

Una vez analizados los factores con los resultados de estudios previos (Aaker, 1997; Goñi et al., 2013; Olavarrieta et al., 2004), se nombraron las dimensiones de la siguiente manera de acuerdo a los factores del primero al octavo: Competencia, Sinceridad, Entusiasmo, Sofisticación, Delicadeza, Rudeza, Regionalismo y Cooperación. Posteriormente se calculó la fiabilidad del instrumento tanto para la escala de manera general, como para cada una de las ocho dimensiones que integran la variable de personalidad de marca; esto se realizó mediante el estadístico alfa de Cronbach $(\alpha)$. El coeficiente para la escala general fue aceptable $(\alpha=0,95)$, al igual que los obtenidos para las dimensiones de Competencia $(\alpha=0,90)$, Sinceridad $(\alpha=0,80)$, Entusiasmo $(\alpha=0,81)$, Sofisticación $(\alpha=$ $0,81)$, Delicadeza $(\alpha=0,71)$, Rudeza $(\alpha=0,70)$, Regionalismo $(\alpha=0,70)$ y Cooperación $(\alpha=0,63)$, todos ellos mostrando valores admisibles sobre la consistencia interna, superiores al límite inferior permitido y sugerido de 0,60 (Malhotra, 2004; Martínez et al., 2006).

El procedimiento general para el diseño del instrumento de medición, así como para su aplicación y análisis de los datos fue el siguiente:

1) Se realizó una búsqueda de información relativa al tema de investigación, indagando en bases de datos electrónicas y revistas científicas.

2) Se elaboró el instrumento tomando como referencia a Aaker (1997) y Goñi et al. (2013), esto por su similitud en los contenidos y demás rasgos que son objeto de medición del presente estudio.

3) Se tradujeron del idioma inglés al español los rasgos originales de la variable personalidad de marca utilizados en el estudio de referencia de Aaker, esto a fin de asegurar que cada ítem fuese claro y de fácil comprensión.

4) Se efectuó la validación por expertos, esto con objeto de garantizar la medición de los rasgos propios del estudio original.

5) Se generó la versión final del instrumento para el desarrollo de la prueba piloto, en la que participaron 10 estudiantes universitarios que no señalaron problema o dificultad alguna con relación a la comprensión de cada uno de los ítems.

6) Se llevó a cabo la aplicación definitiva del instrumento de medición, administrándose de manera voluntaria a todos los estudiantes posibles de preparatoria y universidad.

7) Se capturó la información obtenida en una base de datos del software estadístico SPSS (versión 22), permitiendo con ello efectuar los análisis estadísticos correspondientes -la técnica fue el análisis de correspondencia-.

8) Se redactaron los hallazgos del estudio en los documentos pertinentes para su comunicación y divulgación. 


\section{RESULTADOS}

Para la determinación de la personalidad de marca de las tres universidades o instituciones de educación superior, se consideraron las ocho dimensiones previamente obtenidas en el análisis factorial exploratorio; y éstas, a su vez se conformaron de los siguiente rasgos: Competencia (Inteligente, Confiable, Sincero, Independiente, Especializado, Trabajador, Líder, Corporativo, Seguro, Confiado y Tranquilo); Sinceridad (Realista, Honesto, Saludable y Alegre); Entusiasmo (Único, Actual, Original e Imaginativo); Sofisticación (Exitoso, Clase alta, Encantador y Elegante); Delicadeza (Sentimental, Femenino, Amistoso y Excitante); Rudeza (Atrevido, Rudo, Fuerte y Masculino); Regionalismo (Pueblerino y Norteño); y Cooperación (Deportivo y
Orientado a la familia). Mediante un análisis de correspondencia efectuado entre los rasgos obtenidos $\mathrm{y}$ las universidades, fue posible identificar una asociación significativa reportándose un coeficiente de correlación de 0,03 , además de un estadístico chicuadrada de 219,99 con un valor $p$ menor a 0,001 .

En la Figura 2 es posible percibir cierta asociación entre agrupamientos de determinados rasgos de la personalidad de marca, como por ejemplo: Saludable, Confiable y Sincero, además de Líder, Exitoso y Seguro. Por su parte, en cuanto a las universidades en estudio, los rasgos que las distinguen respectivamente son diferentes, por lo que la distancia entre estas dentro de la gráfica es proporcional y sugiere poca relación o asociación entre las mismas.

Figura 2. Gráfico de relación entre rasgos de personalidad de marca y universidades.

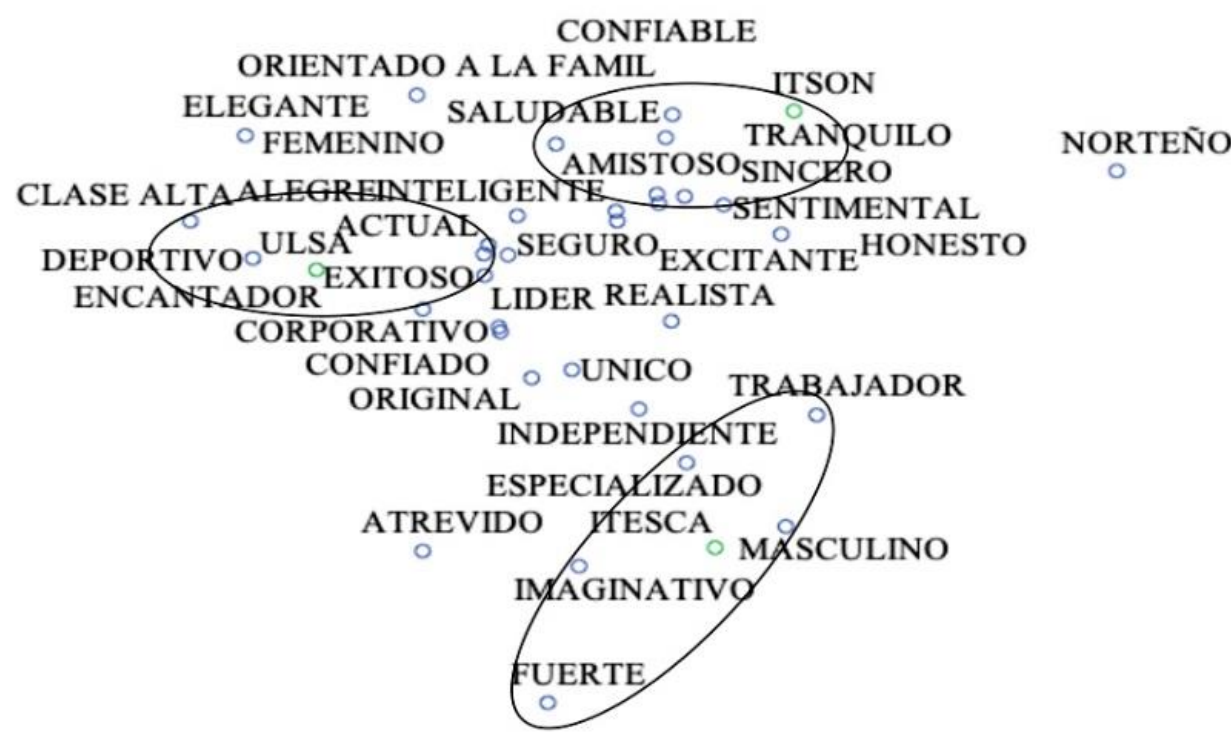

RUDO

O

Fuente: elaboración propia.

Asimismo, se aprecian notables diferencias entre los rasgos más cercanos o asociados a cada institución.
Para el caso de ITSON, los rasgos que presentaron mayor asociación con esta universidad son: 
Femenino, Saludable, Tranquilo, Confiable, Sincero y Sentimental. Así también, a pesar de poseer un menor grado de relación, esta institución fue la más cercana a los rasgos Norteño y Pueblerino, los cuales se encuentran aún más distantes de las otras dos instituciones universitarias. En cuanto al logotipo de la universidad ULSA, los rasgos más asociados con esta institución fueron: Deportivo, Exitoso, Clase alta, Líder y Actual. Finalmente, los rasgos o atributos de Masculino, Imaginativo y Especializado se vincularon en mayor medida con la universidad ITESCA, y un poco más distante con Trabajador y Fuerte. Por último, también es posible apreciar que el rasgo de Rudo se encuentra distante de las tres instituciones, siendo ITESCA la institución más cercana.

Al realizar el análisis de correspondencia efectuado entre las dimensiones obtenidas y las universidades, también se identificó una asociación significativa mostrando un coeficiente de correlación de 0,01, además de un estadístico chi-cuadrada de 61,62 y un valor p menor a 0,001 . Siguiendo un mismo orden en cuanto a la presentación de los hallazgos, en la Figura
3 se muestran ciertas asociaciones entre las dimensiones de la personalidad de marca, siendo posible apreciar una relación entre los factores de Cooperación y Sofisticación, así como de Delicadeza y Sinceridad, y en menor medida entre las dimensiones Competencia y Entusiasmo. Cabe señalar que las dimensiones de Regionalismo y Rudeza fueron las que se encuentran mayormente distantes en comparación a las demás.

En relación a la asociación entre la dimensión de personalidad de marca y las universidades, se observó que las dimensiones de Sinceridad y Delicadeza se asociaron en mayor medida a ITSON, mientras que las de Sofisticación y Cooperación fueron más cercanas a ULSA, siendo a su vez la dimensión denominada como Entusiasmo la que más se relacionó con el logotipo de ITESCA. Estos resultados se originaron con base en la selección de las dimensiones de personalidad de marca que obtuvieron el mayor número de rasgos distintivos de cada institución.

Figura 3. Gráfico de relación entre dimensiones de personalidad de marca y universidades.

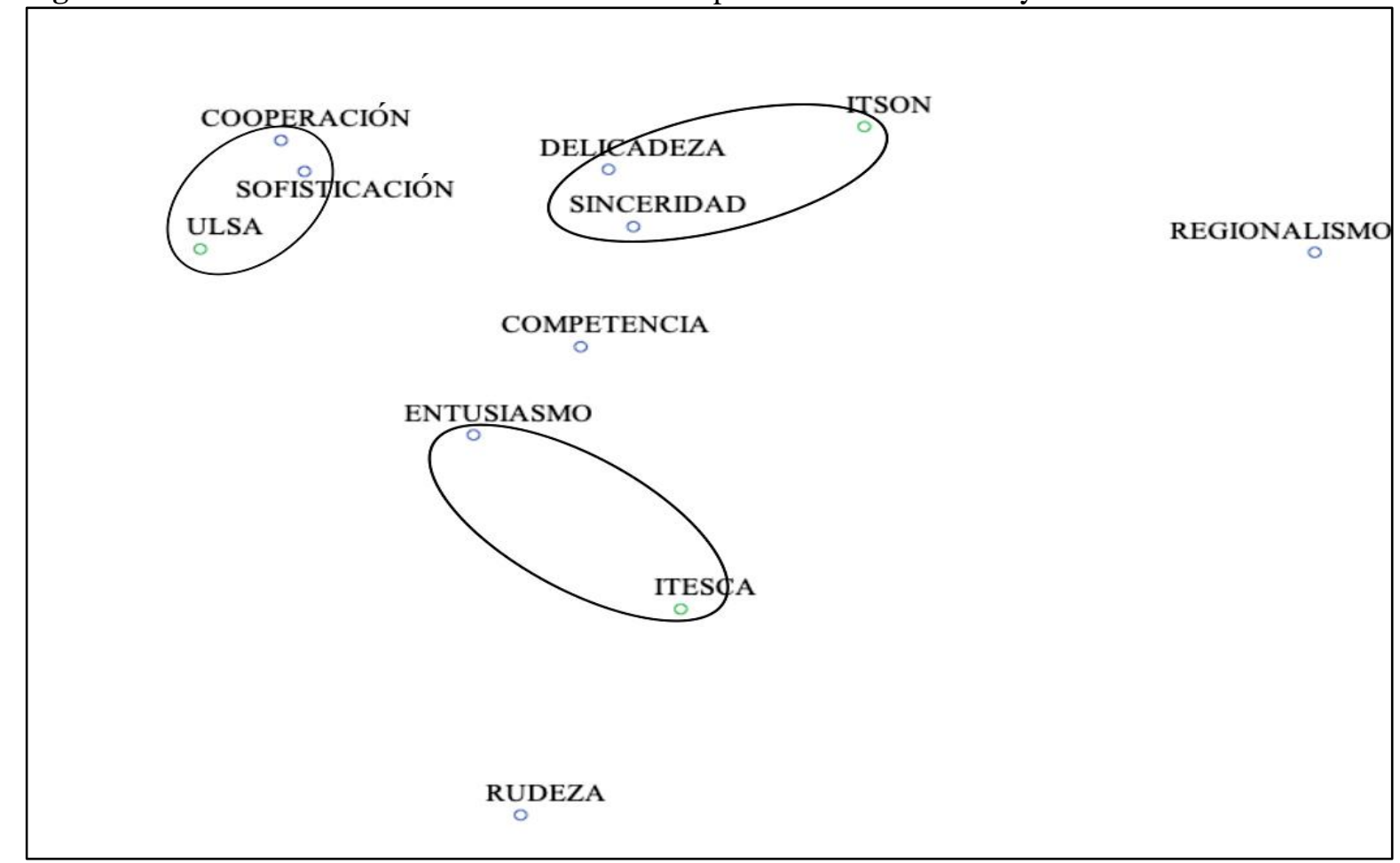

Fuente: elaboración propia. 
Finalmente, se proyectó un análisis de correspondencia entre una dimensión de personalidad de marca ideal -representada por un punto idóneo ubicado en una posición acorde a los atributos de mayor importancia en cada una de las dimensiones- y las universidades, obteniendo una asociación significativa reportando un coeficiente de correlación de 0,01 , además de un estadístico chicuadrada de 61,62 y un valor $p$ menor a 0,001 . Con relación a esto, se aprecia que las tres universidades se encuentran equidistantes con este punto ideal.
De acuerdo con su proximidad o cercanía al escenario idóneo, se asume el siguiente orden de las dimensiones: Competencia, Sinceridad, Entusiasmo, Delicadeza, Sofisticación, Cooperación, Rudeza y Regionalismo (Figura 4).

Debido a lo anterior, puede señalarse que si las instituciones en estudio pretendieran aproximarse al punto idóneo, habría que tomar medidas que fortalezcan la percepción de los rasgos que conforman principalmente la dimensión de Competencia.

Figura 4. Gráfico de situación ideal de personalidad de marca para las universidades.

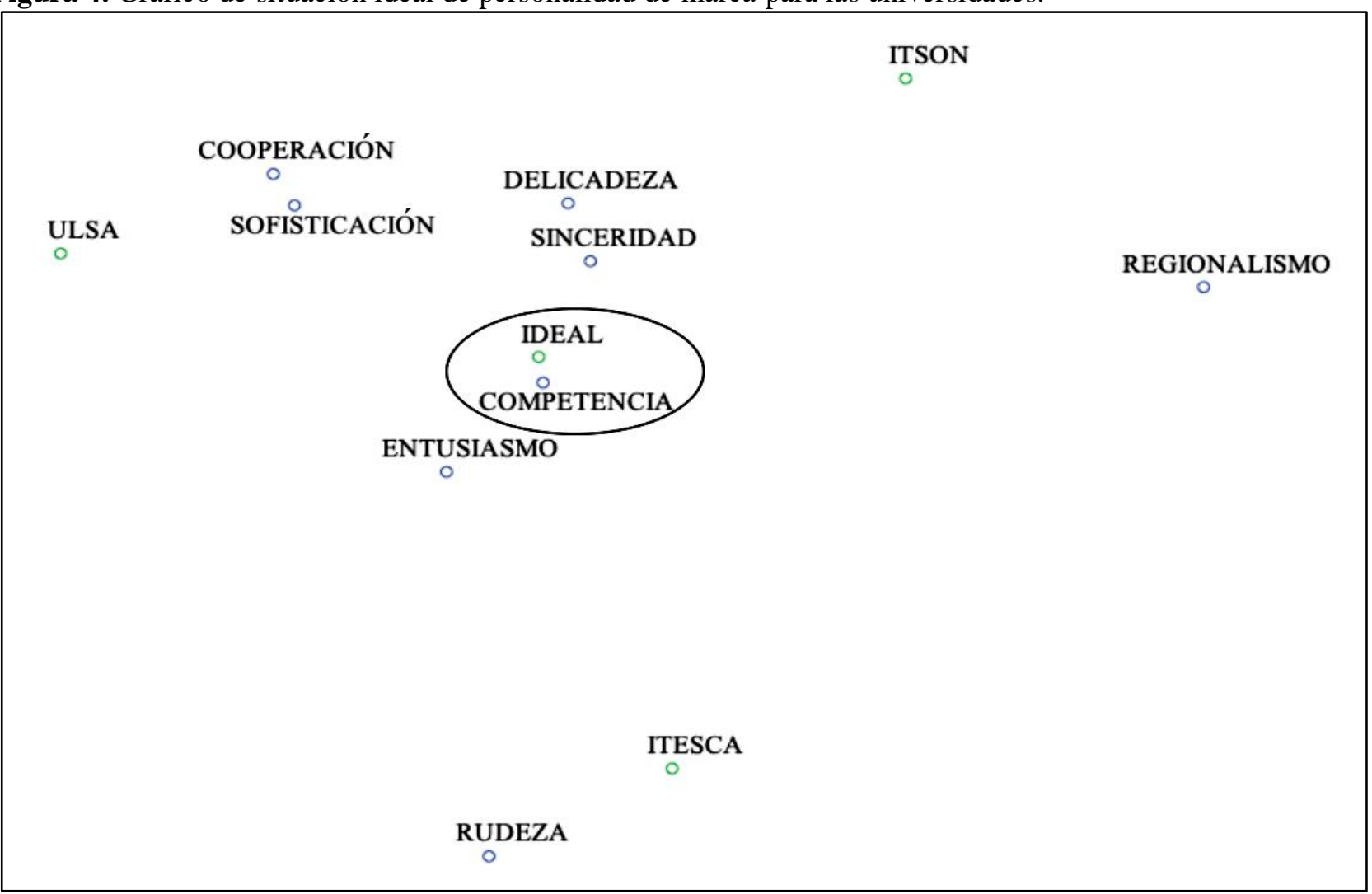

Fuente: elaboración propia.

\section{DISCUSIÓN}

Con relación al trabajo realizado originalmente por Aaker (1997), en el presente estudio se adicionaron tres dimensiones de personalidad de marca: Delicadeza, Regionalismo y Cooperación, lo que representa el principal contraste entre ambas investigaciones. Es posible que esta diferencia se deba a la reagrupación natural de los rasgos evaluados, a efecto de las desemejanzas culturales entre los grupos de personas de cada área geográfica y la diversidad de connotaciones establecidas para cada rasgo. Cabe destacar que en los estudios previos la personalidad de marca se midió considerando numerosos factores, como por ejemplo, la experiencia empírica con los productos o servicios de 
las marcas, así como el conjunto de antecedentes que los sujetos mentalmente poseían de las mismas. Por el contrario, en esta investigación el objeto de apreciación fue únicamente el logotipo de cada institución educativa, considerándolo como elemento transmisor de la personalidad de marca.

$\mathrm{Si}$ bien es complejo determinar el o los motivos subjetivos que llevan a un individuo a emitir cierta percepción sobre algún fenómeno, existen diversos factores que pudiesen influir en este proceso. En el caso particular de la personalidad de marca transmitida por las imágenes de los logotipos institucionales, la psicología del color es considerada como un elemento que interviene en la apreciación de los individuos. Con respecto a este posible factor explicativo, Eva Heller (2008) realizó un estudio donde asocia diferentes colores con numerosos atributos, como sentimientos e impresiones, en los cuales se identificaron algunas similitudes con los resultados de la presente investigación.

En el caso de ITSON, cuyo logotipo se muestra de color azul, se confirma que los rasgos más representativos de esta institución son Femenino, Saludable, Tranquilo, Confiable y Sincero, presentando en diferente medida un cierto grado de asociación con este color. En cuanto a los colores azul y rojo del logotipo de ULSA, se aprecia que tres de los rasgos más característicos de esta universidad fueron Deportivo, Exitoso y Actual, los cuales efectivamente son representados por el color azul. Finalmente, los colores que conforman el logotipo de ITESCA son negro y rojo, los cuales se vinculan con los rasgos de Masculino y Fuerte, característicos de esta institución; además que la combinación específicamente de estos dos colores manifiesta una atribución de "fuerza", reforzando lo previamente mencionado (Heller, 2008, p. 23, 32, 33, 47, 55, 57, 61 y 145).

\section{CONCLUSIÓN}

La presente investigación se propuso medir la percepción de la personalidad de marca de tres instituciones de educación superior en el municipio de Cajeme en México, lo cual a su vez tuvo como finalidad se propicie una adecuada gestión de la marca institucional. Como resultado de los análisis correspondientes se identificaron un total de ocho dimensiones, las cuales se validaron mediante un análisis factorial exploratorio con el objeto de realizar una reducción -simplificación ordenada- de una gran cantidad de ítems o rasgos, en una cantidad menor de factores o constructos representativos. Cabe resaltar el valor metodológico de esta investigación, al existir la posibilidad de replicar el estudio en otras universidades.

Así también, a través de los análisis de correspondencia fue posible determinar una mayor asociación de ciertos rasgos o atributos con cada una de las instituciones educativas. En el caso de ITSON, estos atributos fueron Femenino, Saludable, Tranquilo, Confiable, Sincero y Sentimental; para la ULSA se identificaron Deportivo, Exitoso, Clase alta, Líder y Actual; mientras que para ITESCA los rasgos son Masculino, Imaginativo y Especializado.

En cuanto a las dimensiones resultantes, se observó que la Sinceridad y la Delicadeza poseen mayor asociación con ITSON, las dimensiones de Sofisticación y Cooperación se agruparon con la ULSA, y la dimensión de entusiasmo se vinculó principalmente con ITESCA. Finalmente se proyectó un esquema ideal ubicando un punto de referencia que supone una apreciación idónea, determinando que la dimensión de Competencia es la más valorada por los sujetos de la muestra, dicho hallazgo es similar al obtenido en estudios previos.

Algunas ideas sobre la personalidad de marca que pueden considerarse como áreas de oportunidad para investigaciones posteriores se encuentran las siguientes:

1) Aumentar el número de la muestra para la aplicación del instrumento de medición, al igual que la cantidad de universidades objeto de estudio.

2) Se sugiere conocer de antemano la imagen corporativa que cada institución pretende manifestar con sus logotipos, posibilitando una comparación con los resultados que se obtengan y así contar con material preciso 
para diseñar estrategias oportunas afines a las necesidades de cada entidad.

3) Sería relevante determinar la perspectiva de personalidad de marca de las instituciones desde un grupo o sector alterno, como por ejemplo el empresarial.

4) Finalmente, podría valorarse la personalidad de marca de las universidades desde un enfoque más amplio, en donde además de su logotipo se consideren como indicadores de medición otros elementos institucionales que no se incorporaron en el presente estudio.

Conocer la percepción de la personalidad de marca de diversos grupos de interés y no solo a los consumidores, sin duda proporcionará información estratégica que repercutirá en los objetivos de mercadotecnia al desarrollar mejores materiales de reclutamiento y publicidad y, por tanto, fortalecerá su vínculo con las universidades.

\section{Declaración de conflicto de intereses}

Los autores declaran no poseer ningún conflicto de interés potencial con respecto a la investigación, autoría y/o publicación de este artículo.

\section{Financiamiento}

Los autores declaran no haber recibido apoyo financiero alguno para la investigación, autoría y/o publicación de este artículo.

\section{REFERENCIAS BIBLIOGRÁFICAS}

Aaker, J. L. (1997). Dimensions of Brand Personality. Journal of Marketing Research, 34(3), 347-356. Doi: http://dx.doi.org/10.2307/3151897

Aaker, J. L. (1999). The malleable self: The role of self-expression in persuasion. Journal of Marketing Research, 36(1), 45-57.

Doi: http://dx.doi.org/10.2307/3151914

Aaker, J. L., Benet-Martínez, V. y Garolera, J. (2001). Consumption Symbols as Carriers of Culture: A Study of Japanese and Spanish Brand Personality Constructs. Journal of Personality and Social Psychology, 81(3), 492-508. Doi: http://dx.doi.org/10.1037/00223514.81.3.492

Álvarez-Ortiz, C. y Harris, J. (2002). Assessing the structure of brand personality among global and local Mexican brands. En W. J. Kehoe, y J. H. Lindgren Jr. (Eds.), Enhancing Knowledge Development in Marketing (pp. 263-264). San Diego, CA, USA: Proceedings of the AMA Summer Educator's Conference.

Arpan, L. M., Raney, A. A. y Zivnuska, S. (2003). A cognitive approach to understanding university image. Corporate Communications, 8(2), 97113.

Doi: https://doi.org/10.1108/1356328031047 535

Baños, M. y Rodríguez, T. C. (2012). Imagen de Marca y Product Placement. Madrid, España: ESIC Editorial. Recuperado de https://www.tagusbooks.com/leer?isbn $=9788416701476 \&$ idsource $=3001 \&$ li $=$ 1

Benavides, J. (2012). La investigación en comunicación y publicidad: nuevos temas y problemas. Questiones publicitarias, 1(17), 71-93. Doi: https://doi.org/10.5565/rev/qp.55

Cabezas, D. y Orellana, L. (2014). Estudios de personalidad de marca en educación superior y sectores no tradicionales. Temulco, CL: Universidad de la Frontera. Recuperado de https://www.researchgate.net/profile/M arianela_Denegri_Coria/publication/27 1516317 ESTUDIOS_DE_PERSONA LIDAD_DE_MARCA_EN_EDUCACI ON_SUPERIOR_Y_SECTORES_NO TRADICIONALES/links/54ca6f090c f2c70ce521d578/ESTUDIOS-DEPERSONALIDAD-DE-MARCA-ENEDUCACION-SUPERIOR-Y- 
SECTORES-NO-

TRADICIONALES.pdf

Capriotti, P. (2013). Planificación Estratégica de la Imagen Corporativa (4ta. ed.). Málaga, España: Editorial Ariel. Recuperado de http://www.bidireccional.net/Blog/PEI C 4ed.pdf

Cussi, L. R. (2016). Nivel de relación entre la imagen y la identidad de marca en los estudiantes del año académico 2014 de la Universidad Alas Peruanas en la Ciudad de Tacna (Tesis de maestría). Universidad Nacional Jorge Basadre Grohmann-Tacna, Perú. Recuperado de http://repositorio.unjbg.edu.pe/handle/ UNJBG/1047

Field, A. (2018). Discovering statistics using IBM SPSS statistics (5ta. ed.). London, England: Sage Publications Ltd.

Gómez, A. (2010). Análisis de la estructura de la personalidad de marca y sus efectos sobre la imagen y la lealtad: una aplicación al mercado turístico español (Tesis doctoral). Universidad Autónoma de Madrid, España. Recuperado de https://repositorio.uam.es/handle/10486 15286

Goñi, N., Torres, E. y Aguilera, S. (2013). Dimensiones de la personalidad de la marca en México. Revista de Ciencias Sociales, 19(2), 213-225. Recuperado de

http://www.redalyc.org/articulo.oa?id= 28026992002

Hair, J. F., Anderson, R. E., Tatham, R. L. y Black, W. C. (1999). Análisis Multivariante (5ta. ed.). Madrid, España: Pearson Prentice Hall.

Heller, E. (2008). Psicología del color: Cómo actúan los colores sobre los sentimientos y la razón. Barcelona, España: Editorial Gustavo Gil.

Ho, R. (2006). Handbook of univariate and multivariate data analysis and interpretation with SPSS. Boca Raton, FL, USA: Taylor y Francis Group, LLC.

Keller, K. (1993). Conceptualizing, Measuring, and Managing Customer-Based Brand Equity. Journal of Marketing, 57(1), 122. Doi: https://doi.org/10.2307/1252054

Kleine, S. S., Kleine, R. E. III. y Allen, C. T. (1995). How Is a Possession Me or Not Me? Characterizing Types and an Antecedent of Material Possession Attachment. Journal of Consumer Research, 22(3), 327-343. Doi: https://doi.org/10.1086/209454

Kotler, P. y Armstrong, G. (2013). Fundamentos de marketing (11va. ed.). México: Pearson Educación.

Llopis, E. (2015). Crear la Marca Global: Modelo práctico de creación e internacionalización de marcas. Madrid, España: ESIC Editorial.

Lodos, H. (2011). Lo emocional en las marcas. Open DC - Universidad de Palermo. Buenos Aires, Argentina. Recuperado de

http://www.palermo.edu/dyc/opendc/op endc2011_1/036.pdf

Malhotra, N. K. (1988). Self Concept and Product Choice: An Integrated Perspective. Journal of Economic Psychology, 9(1), 1-28. Doi: https://doi.org/10.1016/01674870(88)90029-3

Malhotra, N. K. (2004). Investigación de mercados: un enfoque aplicado (4ta. ed.). México: Pearson Educación.

Martínez, M. R., Hernández, M. J. y Hernández, M. V. (2006). Psicometría. Madrid, España: Alianza Editorial.

Myers, J. H. y Shocker, A. D. (1981). The nature of product-related attributes. En J. S. Sheth (Ed.), Research in marketing (pp. 211-236). Greenwich, 
UK: JAI Press.

Olavarrieta, S., Friedmann, R. y Manzur, E. (2004). Brand personality outside the developed world: A combined emic-etic study in Chile. En K. L. Bernhardt, J. S. Boles, y P. S. Ellen (Eds.), Enhancing Knowledge Development in Marketing (pp. 390-396). Chicago, USA: American Marketing Association.

Peñaloza, V., Denegri, M., Quezado, I., Campos, E., Barreda, V. A. y Gerhard, F. (2016). Brand personality in psychology courses: a study in cities in Brazil, Peru and Chile. Estudios Gerenciales, 32(140), 239-249. Doi: https://doi.org/10.1016/j.estger.2016.07 .002

Pett, M. A., Lackey, N. R. y Sullivan, J. J. (2003). Making Sense of Factor Analysis: The Use of Factor Analysis for Instrument Development in Health Care Research. California, United States: SAGE Publications Inc.

Pirela, J. L., Villavicencio, H. A. y Saavedra, J. L. (2004). Dimensiones de Personalidad de Marca. Estudio exploratorio en Venezuela. Revista de Ciencias Sociales, 10(3), 430-440. Recuperado de

http://www.redalyc.org/articulo.oa?id= 28010305

Plummer, J. (2000). How personality makes a difference. Journal of Advertising
Research, 40(6), 79-83. Doi: http://dx.doi.org/10.2501/JAR-40-6-79$\underline{83}$

Saavedra, J. L., Urdaneta, D., Pirela, J. L. y Colmenares, O. (2008). Medición de la personalidad de marca en el mercado automotriz. Visión gerencial, 7(1), 183$196 . \quad$ Recuperado de http://www.redalyc.org/pdf/4655/4655 45878009.pdf

Schlesinger, M. W. y Cervera, A. (2008). Estudio comparativo entre personalidad de marca ideal vs. percibida: aplicación a las compañías aéreas. INNOVAR. Revista de Ciencias Administrativas y Sociales, 18(31), 61-75. Recuperado de http://www.redalyc.org/articulo.oa?id= $\underline{81803107}$

Sung, Y. y Tinkham, S. F. (2005). Brand personality structures in the United States and Korea: Common and culturespecific factors. Journal of Consumer Psychology, 15(4), 334-350. Doi: https://doi.org/10.1207/s15327663jcp1 $\underline{504 \_8}$

Watkins, B. A. y Gonzenbach, W. J. (2013). Assessing university brand personality through logos: an analysis of the use of academics and athletics in university branding. Journal of Marketing for Higher Education, 23(1), 15-33. Doi: https://doi.org/10.1080/08841241.2013. $\underline{805709}$ 